\title{
Exosomes Derived from Human Umbilical Cord Mesenchymal Stem Cells Enhance Insulin Sensitivity in Insulin Resistant Human Adipocytes*
}

\author{
Mei-ting CHEN ${ }^{1,2}$, Yi-ting ZHAO ${ }^{3}$, Li-yuan ZHOU ${ }^{1}$, Ming LI $^{1}$, Qian ZHANG ${ }^{1}$, Qin $\mathrm{HAN}^{4}$, Xin-hua XIAO ${ }^{1 \#}$ \\ ${ }^{I}$ Department of Endocrinology, Key Laboratory of Endocrinology, Ministry of Health, Peking Union Medical College Hospital, \\ Diabetes Research Center of Chinese Academy of Medical Sciences \& Peking Union Medical College, Beijing 100730, China \\ ${ }^{2}$ Department of Medical Oncology, Sun Yat-sen University Cancer Center, State Key Laboratory of Oncology in South China, \\ Collaborative Innovation Center for Cancer Medicine, Guangzhou 510060, China \\ ${ }^{3}$ Department of PET-CT Center, Cancer Hospital, Chinese Academy of Medical Sciences \& Peking Union Medical College, \\ Beijing 100021, China \\ ${ }^{4}$ Center of Excellence in Tissue Engineering, Key Laboratory of Beijing, Institute of Basic Medical Sciences and School of Basic \\ Medicine, Chinese Academy of Medical Sciences and Peking Union Medical College, Beijing 100730, China
}

(C) The Author(s) 2021

\begin{abstract}
Summary: Insulin resistance is an essential characteristic of type 2 diabetes mellitus (T2DM), which can be induced by glucotoxicity and adipose chronic inflammation. Mesenchymal stem cells (MSCs) and their exosomes were reported to ameliorate T2DM and its complications by their immunoregulatory and healing abilities. Exosomes derived from MSCs contain abundant molecules to mediate crosstalk between cells and mimic biological function of MSCs. But the role of exosomes derived from human umbilical cord mesenchymal stem cells (hUC-MSCs) in insulin resistance of human adipocytes is unclear. In this study, exosomes were harvested from the conditioned medium of hUC-MSCs and added to insulin-resistant adipocytes. Insulin-stimulated glucose uptake was measured by glucose oxidase/peroxidase assay. The signal pathway involved in exosome-treated adipocytes was detected by RT-PCR and Western blotting. The biological characteristics and function were compared between hUC-MSCs and human adipose-derived mesenchymal stem cells (hAMSCs). The results showed that hAMSCs had better adipogenic ability than hUC-MSCs. After induction of mature adipocytes by adipogenesis of hAMSC, the model of insulin-resistant adipocytes was successfully established by TNF- $\alpha$ and high glucose intervention. After exosome treatment, the insulin-stimulated glucose uptake was significantly increased. In addition, the effect of exosomes could be stabilized for at least $48 \mathrm{~h}$. Furthermore, the level of leptin was significantly decreased, and the mRNA expression of sirtuin-1 and insulin receptor substrate-1 was significantly upregulated after exosome treatment. In conclusion, exosomes significantly improve insulin sensitivity in insulin-resistant human adipocytes, and the mechanism involves the regulation of adipokines.
\end{abstract}

Key words: type 2 diabetes mellitus; insulin resistance; hyperglycemia injury; mesenchymal stem cells; exosome

Type 2 diabetes mellitus (T2DM) is characterized by insulin resistance and is one of the major diseases that endanger human health worldwide ${ }^{[1]}$. Chronic low-

Mei-ting CHEN, E-mail: chenmt@sysucc.org.cn "Corresponding author, E-mail: xiaoxh2014@vip.163.com ${ }^{*}$ This work was supported by the grants from National Key Research and Development Program of China (2016YFA0101002), National Key R\&D Program of China (2017YFC1309603), and National Natural Science Foundation of China (Nos. 81170736, 81570715, 81870579). Electronic supplementary material The online version of this article (https://doi.org/10.1007/s11596-021-2323-4) contains supplementary materials, which are available to authorized users. grade inflammation and hyperglycemic toxicity are important causes of insulin resistance in the adipose tissue of T2DM patients. Further studies reported that the downregulation of adiponectin and insulin receptor substrate (IRS)-1 phosphorylation, as well as the upregulation of interleukin (IL)-6, leads to insulin resistance ${ }^{[2]}$. However, most studies were based on 3T3-L1 cells, and few studies have focused on human adipocytes. Ronningen et al used adipocytes from human adipose-derived mesenchymal stem cells (hAMSCs), induced high glucose damage, and found that increased expression of monocyte chemotactic protein (MCP)-1 and IL-1 led to insulin resistance ${ }^{[3]}$. Compared with the individual effects of chronic 
inflammation and hyperglycemia, the interactions of various factors in adipose tissue were closely related to the disease $\mathrm{e}^{[4,5]}$. Therefore, to better simulate the disease state in vivo, many studies have combined induction agents, such as tumor necrosis factor (TNF)- $\alpha$ plus a hypoxic environment, to establish an insulin-resistant mode ${ }^{[6]}$. In this study, low concentrations of TNF- $\alpha$ and high glucose medium were used to simulate insulin resistance in human adipocytes.

MSCs are nonhematopoietic stem cells with multidirectional differentiation potential that can be extracted from the bone marrow, adipose tissue and umbilical cord ${ }^{[7]}$. Several studies have reported that MSCs coculture or in vivo infusion can improve $\mathrm{T}_{2} \mathrm{DM}^{[8,9]}$. Cell-free therapy, such as conditioned media and exosomes derived from MSCs, has become a research hotspot in recent years. Exosomes are microparticles of $30 \mathrm{~nm}$ to $100 \mathrm{~nm}$ in diameter that are secreted via endocytosis ${ }^{[10]}$. They can be obtained from conditioned medium of MSCs by ultracentrifugation and ultrafiltration, and these microparticles mimic the biological functions of $\mathrm{MSCs}^{[1]}$. Previous studies have confirmed the regenerative effects of MSC-derived exosomes in the treatment of type 1 $\mathrm{DM}^{[12]}$. Exosomes also showed therapeutic benefits in hyperglycemia-induced erectile dysfunction by suppressing inflammatory factors ${ }^{[13,14]}$. Exosomes from MSCs improve insulin sensitivity and reduce obesity via macrophage crosstalk in obese mice ${ }^{[15]}$. However, the exosomes in these studies were all obtained from adipose tissue, which might be affected by the donors. Therefore, our study compared the characteristics and biological functions of hAMSCs and umbilical cordderived MSCs (hUC-MSCs) and explored the role of hUC-MSC-derived exosomes in the insulin sensitivity of adipocytes.

\section{MATERIALS AND METHODS}

\subsection{MSCs Culture and Identification}

hAMSCs were isolated from discarded adipose tissue from patients who underwent liposuction in the Orthopedic Department of Peking Union Medical College Hospital (PUMCH). hUC-MSCs were isolated from the umbilical cords and placentas of healthy pregnant women at PUMCH. Because the procedures did not cause additional harm to the patients and did not reveal personal information, the Ethics Committee of the Institute of Basic Research Medicine of the Chinese Academy of Medical Sciences approved the informed consent exemption. All procedures were in accordance with the ethical standards of the responsible committee on human experimentation (institutional and national) and with the Helsinki Declaration of 1975, as revised in 2000 .

Both hAMSCs and hUC-MSCs were cultured in low-glucose Dulbecco's modified Eagle's medium (DMEM)/F12 (Corning, USA) containing 10\% fetal bovine serum (FBS) (Gibco, USA) and $100 \mathrm{U} / \mathrm{mL}$ penicillin/streptomycin at $37^{\circ} \mathrm{C}$ in a $5 \% \mathrm{CO}_{2}$ incubator. MSCs between the fourth and sixth passages were collected for cell infusion. MSCs (6th passage) were stained with FITC-conjugated antibodies against CD29, CD31, CD34, CD44, CD73, CD90, CD106 and HLADR (1:500; BD Pharmingen, USA) at room temperature for $30 \mathrm{~min}$ in the dark. The immunophenotype of MSCs was assessed by flow cytometry (Accuri CFlow, USA). The multipotency of hUC-MSCs was confirmed by osteogenic and adipogenic differentiation, as indicated by Oil Red $\mathrm{O}$ and Alizarin Red staining, respectively.

\subsection{Exosome Isolation and Identification}

hUC-MSC-derived exosomes were isolated from hUC-MSC-conditioned media. No serum was used in the preparation of the conditioned media. The conditioned media were centrifuged at 3000 $\mathrm{g}$ for $30 \mathrm{~min}$ to remove cell debris. The supernatant was subjected to filtration with a $0.1-\mathrm{mm}$ pore polyethersulfone membrane filter (Corning, USA) to remove cell debris and large vesicles, followed by concentration with a $100000 \mathrm{MW}$-cutoff membrane (CentriPlus-70, Millipore, USA). The supernatant was then ultracentrifuged at $100000 \mathrm{~g}$ for $1 \mathrm{~h}$ at $4^{\circ} \mathrm{C}$ using a 70Ti rotor (Beckman Coulter, USA). The resulting pellets were resuspended in $6 \mathrm{~mL}$ of phosphatebuffered saline (PBS) and ultracentrifuged at 100000 $\mathrm{g}$ for $1 \mathrm{~h}$ at $4^{\circ} \mathrm{C}$ using a 100Ti rotor (Beckman Coulter, USA). Purified exosomes were filtered with a $0.22 \mu \mathrm{m}$ filter before storage at $-80^{\circ} \mathrm{C}$.

Exosomes were identified by the transmission electron microscopy at $80 \mathrm{kV}$. Purified exosomes were fixed with $1 \%$ glutaraldehyde in PBS ( $\mathrm{pH}$ 7.4). After being rinsed, a $20-\mu \mathrm{L}$ drop of the suspension was loaded onto a formvar/carbon-coated grid, negatively stained with $3 \%(\mathrm{w} / \mathrm{v})$ aqueous phosphotungstic acid for $1 \mathrm{~min}$, and observed by the transmission electron microscopy. Exosome-specific markers, including CD63, tumor susceptibility 101 (TSG101) and heat shock protein 90 (Hsp90), as well as the intracellular protein marker calnexin, were identified by Western blotting.

\subsection{Quantitative Real Time-Polymerase Chain Reaction (qRT-PCR)}

Cultured cells were lysed with TRIzol reagent (Invitrogen, USA), and RNA was extracted according to the manufacturer's instructions. One microgram of total RNA from each sample was reverse-transcribed using Moloney Murine Leukemia Virus (M-MLV; Takara, Japan) in a final volume of $20 \mu \mathrm{L}$. PCR amplification was carried out using a Step-one system (Bio-Rad, USA) with SYBR Green master mix (Takara, Japan). All qRT-PCR analyses were carried out in duplicate and normalized to GAPDH. The primers for the related genes are listed in table 1. GAPDH mRNA was used as 
Table 1 Primers of real-time PCR

\begin{tabular}{lll}
\hline Genes (human) & Forward primer $\left(5^{\prime}-3^{\prime}\right)$ & Reverse primer $\left(5^{\prime}-3^{\prime}\right)$ \\
\hline Adiponectin & GGCAGGAAAGGAGAACCTGG & AGCCTTGTCCTTCTTGAAGAG \\
Leptin & TGCCTTCCAGAAACGTGATCC & CTCTGTGGAGTAGCCTGAAGC \\
IL-6 & CCTGAACCTTCCAAAGATGGC & TTCACCAGGCAAGTCTCCTCA \\
IL-1 & AGCTACGAATCTCCGACCAC & CGTTATCCCATGTGTCGAAGAA \\
MCP-1 & CAGCCAGATGCAATCAATGCC & TGGAATCCTGAACCCACTTCT \\
IRS-1 & CAGCATCAGTTTCCAGAAGC & TGAGGTCCTAGTTGTGAATCATG \\
SIRT-1 & TAGCCTTGTCAGATAAGGAAGGA & ACAGCTTCACAGTCAACTTTGT \\
GAPDH & GGTCACCAGGGCTGCTTTTA & GATCTCGCTCCTGGAAGATG \\
\hline
\end{tabular}

IL-6: interleukin 6; IL-1: interleukin 1; MCP-1: monocyte chemotactic protein 1; IRS-1: insulin receptor substrate 1; SIRT-1: sirtuin 1

an internal control.

\subsection{Western Blotting}

After being washed twice with cold PBS, adipocytes were lysed in radioimmunoprecipitation assay (RIPA) lysis buffer (Beyotime, China) with $1 \mathrm{mmol} / \mathrm{L}$ phenylmethylsulfonyl fluoride (PMSF) and protease inhibitor cocktail on ice for $30 \mathrm{~min}$, manually scraped from the culture plates and then quantified using a bicinchoninic acid (BCA) protein assay kit (Beyotime, China). Proteins were separated on $10 \%$ sodium dodecyl sulfate-polyacrylamide gel electrophoresis (SDS-PAGE) gels and electroblotted onto a polyvinylidene difluoride (PVDF) membrane (0.22 $\mu \mathrm{m}$, Millipore, USA). The membranes were blocked with $5 \%$ bovine serum albumin (BSA), incubated with specific antibodies overnight at $4{ }^{\circ} \mathrm{C}$ and then incubated with horseradish peroxidase (HRP)conjugated secondary antibodies for $1 \mathrm{~h}$ at room temperature. Antibodies against IRS-1, adiponectin, IL-1 $\beta$, CD63, Hsp90 and $\beta$-actin were purchased from Sanying Biological Company (China). The TSG101 antibody was purchased from Abcam (UK). Secondary HRP-conjugated antibodies were from NeoBioscience (China). Antibody, and antigen complexes were detected using electrochemiluminescence reagents (Millipore, USA). $\beta$-actin protein expression was used as an internal control.

\subsection{Measurement of Insulin-stimulated Glucose Uptake}

After adipogenic induction of hAMSCs, mature adipocytes were obtained. Insulin resistance was induced by the administration of $10 \mathrm{ng} / \mathrm{mL}$ TNF- $\alpha$ for $48 \mathrm{~h}$ and cultured in high glucose medium ( 25 and 35 $\mathrm{mmol} / \mathrm{L}$ glucose) for $24 \mathrm{~h}$. After serum starvation in low glucose DMEM overnight, $10 \mathrm{ng} / \mathrm{mL}$ insulin was added to the starved adipocytes. The glucose uptake assay was performed by the glucose oxidase/peroxidase (GOPOD) method (GOPOD assay kit, Megazyme International, Ireland) according to the manufacturer's instructions. Residual glucose in the cell media was determined by measuring the absorbance $(A)$ at $510 \mathrm{~nm}$ (Biotek Synergy, USA). Based on the obtained value of the residual glucose, the percentage of glucose uptake was calculated.

\subsection{Statistical Analysis}

SPSS 19.0 software (SPSS Inc., USA) was used for data processing. A value of $P<0.05$ was required for the results to be considered statistically significant. The data were presented as the mean \pm standard error of the mean and determined by Student's $t$ test or ANOVA followed by a post SNK $q$ test as appropriate.

\section{RESULTS}

\subsection{Characterization of MSCs and Exosomes}

Both hAMSCs and hUC-MSCs were fusiform and fibroblast-like (supplemental fig. 1A and 1B). The immunotypes of 6th-passage hAMSCs and hUCMSCs were analyzed by flow cytometry (supplemental fig. 1C and 1D). hAMSCs exhibited high expression of CD29, CD44 and CD73, as well as negative expression of CD31, CD106 and HLA-DR, while hUC-MSCs showed high expression of CD44 and CD90 and negative expression of CD31, CD34, CD106 and HLA-DR, exhibiting the typical molecular profile of MSCs. The multilineage differentiation of MSCs was compared by osteogenic and adipogenic induction (supplemental fig. 1E-1H). Using Oil Red O staining to confirm adipogenesis, hAMSCs exhibited large, round cells with cytoplasmic lipid-rich vacuoles (supplemental fig. 1E), while a few hUC-MSCs displayed intracellular lipid droplets (supplemental fig. 1F). For osteogenic differentiation, extracellular matrix mineralization was visualized by Alizarin Red S staining. No differences in osteogenesis were observed between hAMSCs (supplemental fig. 1G) and hUCMSCs, but hUC-MSCs were lightly stained after Alizarin Red staining (supplemental fig.1H).

After being cultured for $48 \mathrm{~h}$ in serum-free medium, the cell supernatant was collected, and exosomes from hUC-MSCs were obtained by ultrafiltration. Transmission electron microscopy showed that a large number of round microvesicles with deep outer membranes were observed, and the diameters were approximately 30-100 nm (fig. 1A). Western blot analysis indicated that these microvesicles positively expressed the exosome marker proteins CD63, Hsp90, and TSG101 and did not express calnexin (fig. 1B). 


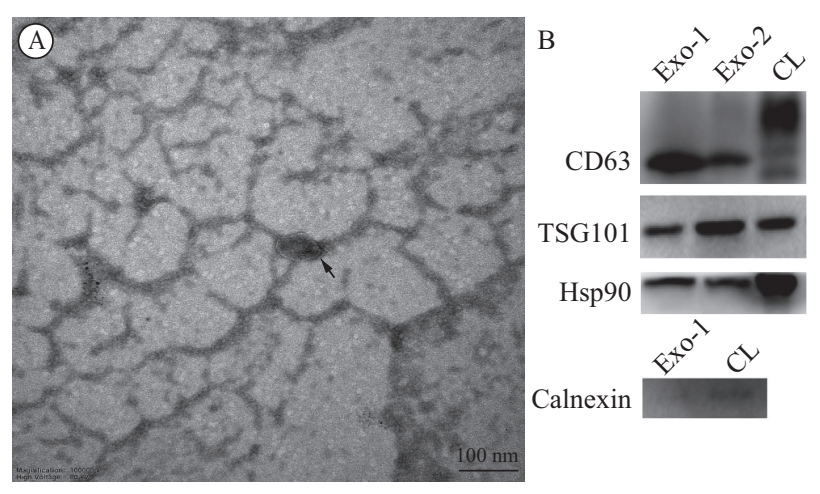

Fig. 1 Identification of exosomes from MSCs

A: electron microscopy analysis of exosomes (indicated by the black arrow) secreted by hUC-MSCs. Scale bar $=100 \mathrm{~nm}$. B: Western blot analysis of the exosomespecific marker TSG101, extracellular vesicle-related protein markers Hsp90 and CD63, intracellular protein marker calnexin. Two samples of exosomes from replicated independent experiments were analyzed. Exo: exosome; CL: cell lysis

\subsection{Insulin Resistance Model in Human Adipocytes}

After adipogenic induction, the induction media were replaced with basal media (DMEM $+10 \%$ FBS). Then, hAMSCs were divided into 6 groups. The concentration of glucose in the culture media in the control group was $4 \mathrm{mmol} / \mathrm{L}$ without TNF- $\alpha$ administration. For the hyperglycemia groups, 25,30 , and $35 \mathrm{mmol} / \mathrm{L}$ glucose was added to the media. In the inflammation group, $10 \mathrm{ng} / \mathrm{mL}$ TNF- $\alpha$ was added. We also established a hyperglycemia plus chronic inflammation group, in which $10 \mathrm{ng} / \mathrm{mL}$ TNF- $\alpha$ combined with $35 \mathrm{mmol} / \mathrm{L}$ glucose was administered. The results of the insulinstimulated glucose uptake experiments are shown in supplemental fig. 2A. After the cells were stimulated with high glucose and TNF- $\alpha$ for $24 \mathrm{~h}$, insulin was used to induce glucose uptake, and the expression of inflammatory factors and adipokines was analyzed in each group (supplemental fig. 2B-2H). Compared with that of the control group, glucose uptake after insulin stimulation was significantly decreased in the hyperglycemia group and inflammation plus hyperglycemia group $(P<0.05)$. After the induction of insulin resistance, the expression of proinflammatory cytokines (MCP-1, IL-1, and IL-6) was significantly upregulated $(P<0.05)$, while the mRNA levels of adiponectin and IRS-1 decreased $(P<0.05)$. Leptin expression in the 30 and $35 \mathrm{mmol} / \mathrm{L}$ hyperglycemia groups increased significantly $(P<0.05)$. Adiponectin protein expression was downregulated and IL-1 protein expression was upregulated in the inflammation plus hyperglycemia group. However, no significant difference in IRS-1 protein expression was observed.

2.3 hUC-MSC-derived exosomes Enhance Insulin Sensiti-vity in Adipocytes with Chronic Inflammation and Hyperglycemic Injury

After establishing the insulin resistance model, we treated insulin-resistant adipocytes with $20 \mu \mathrm{g} /$ $\mathrm{mL}$ exosomes for $72 \mathrm{~h}$. Glucose uptake experiments after insulin stimulation and exosome treatment were performed. Compared with that of control group, the insulin-stimulated glucose uptake after exosome treatment was significantly increased $(P<0.05$, fig. 2 and supplemental fig. $3 \mathrm{~A}-3 \mathrm{C}$ ), indicating that exosome treatment could significantly improve insulin sensitivity in insulin-resistant human adipocytes.

To investigate whether the effects of exosomes were stable, we repeated the glucose uptake experiment on the second day. It was clear that glucose uptake remained significantly increased after exosome treatment, and the trend was similar on the second day as shown in fig. 2 and supplemental fig. 3D-3F. Therefore, the therapeutic effect of exosomes was stable for at least $48 \mathrm{~h}$.

\section{4 hUC-MSC-derived Exosomes Upregulate Adipo- nectin and Sirtuin (SIRT)-1 mRNA Expression}

To explore the mechanism of exosome treatment, we compared mRNA and protein expression among the control group, inflammation group, 35 $\mathrm{mmol} / \mathrm{L}$ hyperglycemia group, inflammation plus hyperglycemia group, exosome-treated group and hUC-MSC-treated group. Exosomes were administered in the inflammation group, $35 \mathrm{mmol} / \mathrm{L}$ hyperglycemia group and inflammation plus hyperglycemia group (fig. 3A-3E). hUC-MSCs were cocultured with insulinresistant adipocytes and divided into two groups: the inflammation group with hUC-MSCs and the 35 $\mathrm{mmol} / \mathrm{L}$ hyperglycemia group with hUC-MSCs (fig. $3 \mathrm{~A}-3 \mathrm{E})$. After exosome treatment in the inflammation plus hyperglycemia group, the mRNA expression of adiponectin was significantly increased (fig. 3A). The mRNA levels of leptin after exosome treatment were significantly decreased in the three groups, including the inflammation group, $35 \mathrm{mmol} / \mathrm{L}$ hyperglycemia group and inflammation plus hyperglycemia group (fig. 3B). As shown in fig. 3C and 3D, the mRNA expression levels of both SIRT-1 and IRS-1 were significantly upregulated in the inflammation group and inflammation plus hyperglycemia group after exosome treatment. IRS-1 protein expression was not significantly different among the groups after exosome treatment (fig. 3E). The protein expression of adiponectin was significantly upregulated in the inflammation group after exosome intervention (fig. 3E). In addition, we also evaluated the effect of coculturing hUC-MSCs with insulin-resistant adipocytes, but no significant changes in the mRNA or protein expression patterns of adipokines, IRS-1, and SIRT-1 were observed (fig. 3A-3E).

\section{DISCUSSION}

In our study, we successfully established an 


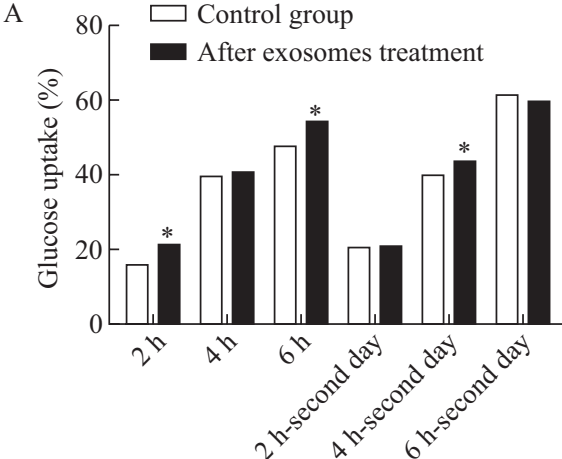

Time after insulin stimulation

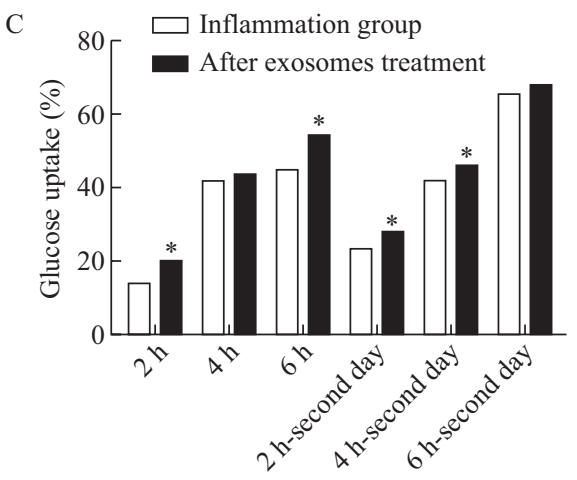

Time after insulin stimulation

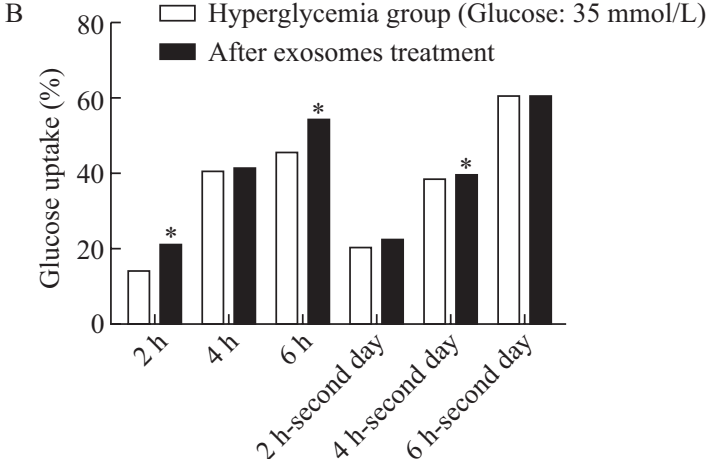

Time after insulin stimulation

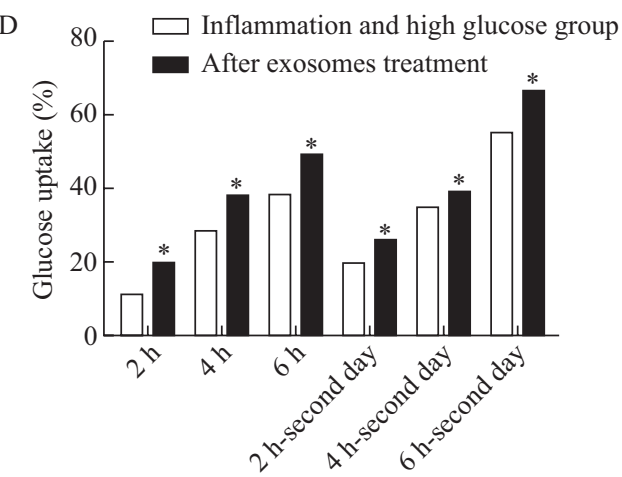

Time after insulin stimulation

Fig. 2 Exosomes improve insulin-stimulated glucose uptake

Glucose uptake was detected in adipocytes by the glucose oxidase/peroxidase (GOPOD) method 2, 4, $6 \mathrm{~h}$ after exosome treatment and experiments repeated on the second day. A: control group; B: hyperglycemia group; C: inflammatory group; D: inflammatory and hyperglycemia group. ${ }^{*} P<0.05 v s$. different control groups

A

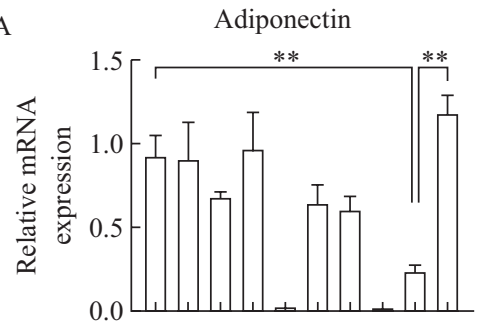

Glucose (mmol/L) 443535354443535

$\mathrm{TNF} \alpha$

Exosomes -+-+--+--+

hUC-MSCs
B

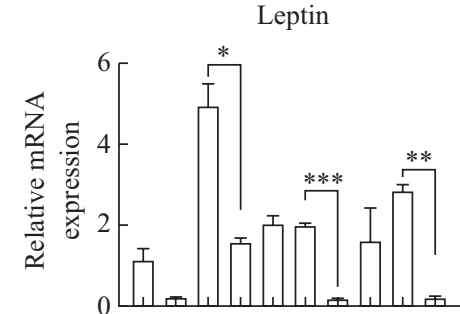

Glucose (mmol/L) 443535354443535

$\mathrm{TNF} \alpha-\ldots+\ldots++++$

Exosomes -+-+--+--+
C

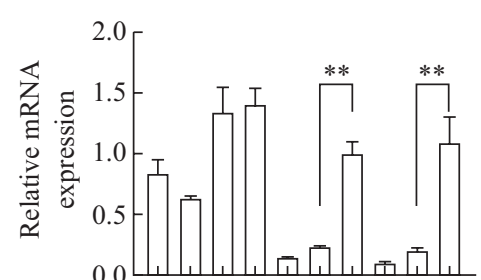

Glucose (mmol/L) 4433535354443535

TNF $\alpha--\ldots-+++++$

Exosomes -+-+-+--+

hUC-MSCs ---++-+-
D

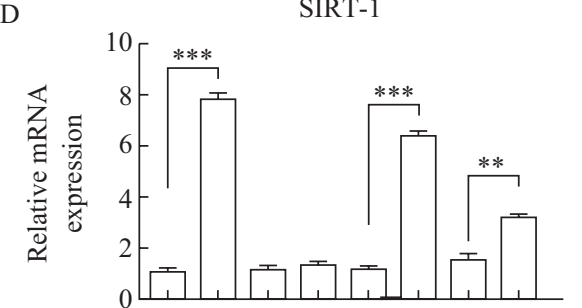

E

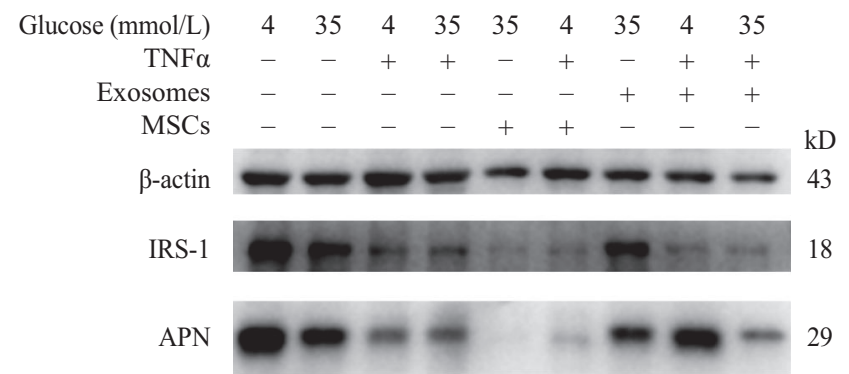

Fig. 3 Mechanism of exosomes improving insulin sensitivity in adipocytes

A-D: the relative expression levels of adiponectin, leptin, IRS-1 and SIRT-1 mRNA in insulin-resistant adipocytes before and after co-culture with exosomes and hUC-MSCs. Reference gene: GAPDH; E: IRS-1, adiponectin protein expression level after co-culture with exosomes and hUC-MSCs. APN: adiponectin, reference gene: $\beta$-actin. ${ }^{*} P<0.05,{ }^{* *} P<0.01,{ }^{* * *} P<0.001$ 
insulin resistance model of hAMSCs. We demonstrated that exosomes derived from hUC-MSCs improved insulin sensitivity in human adipocytes by upregulating adiponectin and SIRT-1 expression. Common sources of MSCs include the bone marrow, adipose tissue and umbilical cord. From these sources, hAMSCs can be obtained and cultured, but they are also easily influenced by the donor and are associated with some ethical problems ${ }^{[16]}$. Compared with other MSCs, hUCMSCs exhibited lower immunogenicity and were easy to obtain, and these cells had broad prospects in tissue engineering applications ${ }^{[17]}$. The differentiation ability of different MSCs varies. Compared with hAMSCs and bone marrow-derived MSCs, hUC-MSCs have limited adipogenic differentiation capacity ${ }^{[7]}$. Our study demonstrated that hAMSCs had better osteogenic and adipogenic abilities than hUC-MSCs. Both hAMSCs and hUC-MSCs exhibited common MSC immunophenotype ${ }^{[18]}$. The 3T3-L1 cell line is the most widely used cell line to establish an insulin resistance model, but the transformation efficiency of 3T3-L1 cells remains low ${ }^{[19]}$. To obtain stable adipocytes, some studies have tried to use hAMSCs to establish models ${ }^{[3]}$. Our study successfully established an insulin resistance model using human adipocytes induced from hAMSCs.

Many studies have noted that exosomes from omental adipose tissue and macrophages are biomarkers and mediators of diabetes and obesity, but exosomes derived from stem cells show beneficial effects for the treatment of diabetic complications ${ }^{[20,21]}$. In our study, hUC-MSC-derived exosomes significantly improved insulin sensitivity in human adipocytes with inflammatory and hyperglycemic injury. Our findings also demonstrated persistent improvements in insulin sensitivity for at least $48 \mathrm{~h}$, indicating a stable interaction between exosomes and target cells. The interaction of exosomes with adipocytes might be a slow process. Exosome uptake and internalization will be analyzed by flow cytometry in future studies.

The mechanism of exosome treatment in diabetes and diabetic complications are under extensive exploration, with various investigations on different signal transduction pathways. In mouse macrophages, exosomes derived from adipose-derived stem cells can induce the production of anti-inflammatory factors to improve metabolic homeostasis ${ }^{[15]}$. In obese mice, the injection of exosomes derived from macrophages from lean mice improved insulin sensitivity by activating peroxisome proliferator-activated receptor (PPAR)- $\gamma^{[22]}$. It is likely that the beneficial effect of exosomes on insulin resistance is related to the regulation of adipocytokines. In our study, the expression of adiponectin and SIRT-1 was upregulated and the expression of leptin was downregulated after exosome treatment. Adipocytokines, including adiponectin, leptin and inflammatory cytokines, play important roles in glucose and lipid metabolism. However, the interaction of adiponectin and exosomes in vivo is complex ${ }^{[23]}$. Exosome biogenesis might be stimulated by serum adiponectin, but the metabolic roles of adiponectin-mediated exosome production are still unknown ${ }^{[24]}$. The expression of leptin in adipocytes promoted insulin resistance by upregulating the expression of proinflammatory factors such as IL-6 and TNF- $\alpha$ and inhibiting the tyrosine phosphorylation of IRS-1 ${ }^{[25]}$. However, the crosstalk between leptin and exosomes is still unclear. In our study, the upregulation of adiponectin and downregulation of leptin might be mediated by exosomes from hUC-MSCs. SIRT-1 is a deacetylase that is widely expressed in various tissues, such as the liver, adipose and skeletal muscle tissue ${ }^{[26]}$. SIRT-1 could induce insulin secretion and activate the expression of IRS-1 to improve insulin sensitivity. Yuan et al found that conditioned medium from MSCs activates phosphoinositide 3-kinase (PI3K) phosphorylation to upregulate SIRT-1 expression and ameliorate vascular endothelial disorders caused by diabetes $^{[27]}$. Therefore, we hypothesize that exosomes derived from hUC-MSCs improve insulin sensitivity via SIRT-1 activation, leading to the downstream regulation of adipocytokines.

However, there were still some limitations in this study. The expression of phosphorylated IRS-1 and PI3K has not been explored. The internalization of exosomes by insulin-resistant adipocytes needs to be investigated to fully understand the interaction of exosomes with target cells. Further research will focus on the regulation of the insulin signal transduction pathway after exosome treatment both in vitro and in vivo.

In summary, hAMSCs were stable and effective seed cells for establishing an adipocyte insulin resistance model. Exosomes derived from hUCMSCs could improve the insulin resistance of human adipocytes. The mechanism might involve upregulating the expression of adiponectin and SIRT1 and downregulating leptin expression. Therefore, exosomes from hUC-MSCs have great therapeutic potential for T2DM patients.

\section{Open Access}

This article is licensed under a Creative Commons Attribution 4.0 International License https://creativecommons.org/licenses/by/4.0/), which permits use, sharing, adaptation, distribution and reproduction in any medium or format, as long as you give appropriate credit to the original author(s) and the source, provide a link to the Creative Commons licence, and indicate if changes were made. The images or other third party material in this article are included in the article's Creative Commons licence, unless indicated otherwise in a credit line to the material. If material is not included in the article's Creative Commons 
licence and your intended use is not permitted by statutory regulation or exceeds the permitted use, you will need to obtain permission directly from the copyright holder. To view a copy of this licence, visit http://creativecommons. org/licenses/by/4.0/.

\section{Conflict of Interest Statement} interest.

The authors have declared that there is no conflict of

\section{REFERENCES}

1 Reusch JE, Manson JE. Management of Type 2 Diabetes in 2017: Getting to Goal. JAMA, 2017,317(10):10151016

2 Zhang Y, Mei HL, Chang XA, et al. Adipocyte-derived microvesicles from obese mice induce M1 macrophage phenotype through secreted miR-155. J Mol Cell Biol, 2016,8(6):505-517

3 Ronningen $\mathrm{T}$, Shah A, Reiner A, et al. Epigenetic priming of inflammatory response genes by high glucose in adipose progenitor cells. Biochem Biophys Res Commun, 2015,467(4): 979-986

4 Lo KA, Kennedy N, Han M, et al. Analysis of in vitro insulin-resistance models and their physiological relevance to in vivo diet-induced adipose insulin resistance. Cell Reports, 2013,5(1):259-270

5 Kupsal K, Mudigonda S, Gundapaneni K, et al. Glucotoxicity and lipotoxicity induced beta-cell apoptosis in type 2 diabetes mellitus. Int J Anal Bio-sci, 2015,3(4):84-89

6 An X, Li L, Chen Y, et al. Mesenchymal Stem Cells Ameliorated Glucolipotoxicity in HUVECs through TSG-6. Int J Mol Sci, 2016,17(4):483

7 Rebelatto C, Aguiar A, Moretão M, et al. Dissimilar differentiation of mesenchymal stem cells from bone marrow, umbilical cord blood, and adipose tissue. Exp Biol Med (Maywood), 2008,233(7):901-913

8 Xie ZY, Hao HJ, Tong C, et al. Human umbilical cordderived mesenchymal stem cells elicit macrophages into an anti-inflammatory phenotype to alleviate insulin resistance in type 2 diabetic rats. Stem Cells, 2016,34(3): 627-639

9 Xie M, Hao HJ, Cheng Y, et al. Adipose-derived mesenchymal stem cells ameliorate hyperglycemia through regulating hepatic glucose metabolism in type 2 diabetic rats. Biochem Biophys Res Commun, 2017,483(1): 435-441

10 Phinney D, Pittenger M. Concise Review: MSCDerived Exosomes for Cell-Free Therapy. Stem Cells, 2017,35(4):851-858

11 Lawson C, Vicencio J, Yellon D, et al. Microvesicles and exosomes: new players in metabolic and cardiovascular disease. J Endocrinol, 2016,228(2):R57-R71

12 Sabry D, Marzouk S, Zakaria R, et al. The effect of exosomes derived from mesenchymal stem cells in the treatment of induced type 1 diabetes mellitus in rats. Biotechnol Lett, 2020,42(8):1597-1610

13 Zhu LL, Huang X, Yu W, et al. Transplantation of adipose tissue-derived stem cell-derived exosomes ameliorates erectile function in diabetic rats. Andrologia, 2018,50(2):e12871

14 Wang J, Mi Y, Wu S, et al. Exosomes from adiposederived stem cells protect against high glucoseinduced erectile dysfunction by delivery of corin in a streptozotocin-induced diabetic rat model. Regenerative therapy, 2020,14:227-233

15 Zhao H, Shang Q, Pan Z, et al. Exosomes from AdiposeDerived Stem Cells Attenuate Adipose Inflammation and Obesity through Polarizing M2 Macrophages and Beiging in White Adipose Tissues. Diabetes, 2018,67: 235-247

16 Moreira A, Kahlenberg S, Hornsby P, et al. Therapeutic potential of mesenchymal stem cells for diabetes. J Mol Endocrinol, 2017,59(3):R109-R120

17 Pan XH, Huang X, Ruan GP, et al. Umbilical cord mesenchymal stem cells are able to undergo differentiation into functional islet-like cells in type 2 diabetic tree shrews. Mol Cell Probes, 2017,34:1-12

18 Kobolak J, Dinnyes A, Memic A, et al. Mesenchymal stem cells: Identification, phenotypic characterization, biological properties and potential for regenerative medicine through biomaterial micro-engineering of their niche. Methods, 2016,99:62-68

19 Vishwanath D, Srinivasan H, Patil MS, et al. Novel method to differentiate $3 \mathrm{~T} 3 \mathrm{~L} 1$ cells in vitro to produce highly sensitive adipocytes for a GLUT4 mediated glucose uptake using fluorescent glucose analog. J Cell Commun Signal, 2013,7(2):129-140

20 Xiao Y, Zheng L, Zou X, et al. Extracellular vesicles in type 2 diabetes mellitus: key roles in pathogenesis, complications, and therapy. J Extracell Vesicles, 2019, 8(1): 1625677

21 Noren H, Evans M. Extracellular vesicles as signaling mediators in type 2 diabetes mellitus. Am J Physiol Cell Physiol, 2020,318(6):C1189-C1199

22 Ying W, Riopel M, Bandyopadhyay G, et al. Adipose Tissue Macrophage-Derived Exosomal miRNAs Can Modulate In Vivo and In Vitro Insulin Sensitivity. Cell, 2017,171(2):372-384

23 Kita S, Maeda N, Shimomura I. Interorgan communication by exosomes, adipose tissue, and adiponectin in metabolic syndrome. J Clin Invest, 2019, 129(10):4041-4049

24 Obata Y, Kita S, Koyama Y, et al. Adiponectin/Tcadherin system enhances exosome biogenesis and decreases cellular ceramides by exosomal release. JCI Insight, 2018,3(8):e99680

25 Könner A, Brüning J. Selective insulin and leptin resistance in metabolic disorders. Cell Metab, 2012, 16(2):144-152

26 Liang F, Kume S, Koya D. SIRT1 and insulin resistance. Nat Revi Endocrinol, 2016,5(7):178-183

27 Yuan Y, Shi M, Li L, et al. Mesenchymal stem cellconditioned media ameliorate diabetic endothelial dysfunction by improving mitochondrial bioenergetics via the Sirt1/AMPK/PGC-1 alpha pathway. Clin Sci (Lond), 2016,130(23):2181-2198

(Received Oct. 7, 2019; accepted Nov. 19, 2020) 